\title{
Co-existence of myopia and amblyopia in a guinea pig model with monocular form deprivation
}

\author{
Lu Tian ${ }^{1,2}$, Ya-Tu Guo ${ }^{1,2}$, Ming Ying ${ }^{1,2}$, Yang-Chen Liu ${ }^{1,2}$, Xuan Li $^{1,2}$, Yan Wang ${ }^{1,2}$ \\ ${ }^{1}$ Clinical College of Ophthalmology, Tianjin Medical University, Tianjin, China; ${ }^{2}$ Tianjin Eye Institute, Tianjin Key Lab of Ophthalmology and \\ Visual Science, Tianjin Eye Hospital, Tianjin, China \\ Contributions: (I) Conception and design: Y Wang, X Li; (II) Administrative support: None; (III) Provision of study materials or patients: None; (IV) \\ Collection and assembly of data: L Tian, YT Guo; (V) Data analysis and interpretation: L Tian, YT Guo; (VI) Manuscript writing: All authors; (VII) \\ Final approval of manuscript: All authors. \\ Correspondence to: Yan Wang, MD, PhD; Xuan Li, MD, PhD. Tianjin Eye Hospital, No. 4. Gansu Rd, He-ping District, Tianjin 300020 , China. \\ Email: wangyan7143@vip.sina.com; xuanli08@yahoo.com.
}

Background: Form deprivation myopia is a type of ametropia, with identifiable causes in humans, that has been induced in many animals. The age of onset of myopia induced by monocular form deprivation coincides with the period of visual development in guinea pigs. However, visual acuity of form-deprived eyes in guinea pigs is not understood yet. In this study, we investigated whether monocular form deprivation would affect visual acuity in infant guinea pigs by evaluating the development of myopia and amblyopia after monocular form deprivation, and whether form deprivation myopia and amblyopia occurred simultaneously or successively.

Methods: Twenty pigmented guinea pigs (2 weeks old) were randomly assigned to two groups: monocularly form-deprived $(\mathrm{n}=10)$, in which facemasks modified from latex balloons covered the right eye, and normal controls ( $n=10)$. Refraction, axial length, and visual acuity were measured at 4 intervals (after $0,1,4$, and 8 weeks of form deprivation), using cycloplegic streak retinoscopy, A-scan ultrasonography (with an oscillation frequency of $10 \mathrm{MHz}$ ), and sweep visual evoked potentials (sweep VEPs), respectively. Sweep VEPs were performed with correction of the induced myopic refractive error.

Results: Longer deprivation periods resulted in significant refractive errors in form-deprived eyes compared with those in contralateral and normal control eyes; the axial lengths of form-deprived eyes increased significantly after 4 and 8 weeks of form deprivation. These results revealed that myopia was established at 4 weeks. The acuity of form-deprived eyes was unchanged compared to that at the pretreatment time point, while that of contralateral eyes and eyes in normal control guinea pigs improved; there were significant differences between the deprived eyes and the other two open eyes from 1 to 8 weeks of form deprivation, showing that amblyopia was possibly established during 1 week of form deprivation.

Conclusions: This study demonstrated the feasibility of using sweep VEPs to estimate the visual acuity of guinea pigs. Further, our results revealed that amblyopia likely occurred earlier than myopia; amblyopia and myopia coexisted after a long duration of monocular form deprivation in guinea pigs. Understanding this relationship may help provide insights into failures of treatment of amblyopia associated with myopic anisometropia.

Keywords: Myopia; amblyopia; form deprivation; guinea pig

Submitted Jul 22, 2020. Accepted for publication Oct 30, 2020.

doi: $10.21037 / \mathrm{atm}-20-5433$

View this article at: http://dx.doi.org/10.21037/atm-20-5433 


\section{Introduction}

Form deprivation myopia is a type of ametropia in humans, with identifiable causes such as congenital cataract, ptosis, and corneal opacity. In order to investigate the mechanisms of myopia in humans, form deprivation myopia has been induced in many animals (1-5). Animal experimental studies have established the importance of visual feedback in eye and refractive state development, and have demonstrated that form deprivation myopia is a graded phenomenon; increases in the degree of image degradation have been positively correlated with the severity of the induced axial myopia, the uncoordinated ocular growth has been found to be due to reduced retinal image contrast, and the absence of visual feedback has been reported to be related to the effective refractive state of the eye (6-8). Many experiments regarding normal visual development in these animals have shown that the age of onset of form deprivation myopia in these animals coincided with the period of visual development, and that larger refractive errors were present in younger animals after a longer duration of form deprivation (9-13). For example, monocular form deprivation in monkeys at the age of 2 weeks and led to the development of myopia of -13.5 diopters (D) after 18 months; monocular form deprivation at the age of 1 year led to the development of only $-4.5 \mathrm{D}$ myopia after 26 months. Eye development in the guinea pigs was basically completed by 3 weeks, and considerable myopia of approximately $-3.4,-5.8$, and $-5.7 \mathrm{D}$ was present after 6,11, and 16 days of form deprivation, respectively, at the age of 5 days. Similarly, any abnormal visual experience (e.g., monocular form deprivation, strabismus, or anisometropia) imposed during the period of visual development could result in abnormalities of the visual system (e.g., amblyopia); however, as this was initiated at progressively older ages, the resulting abnormality was smaller or even without visual impairment at all (14). For example, in rhesus monkeys, monocular form deprivation initiated during early life produced a severe degree of amblyopia, and the effect on spatial vision decreased systematically as the age of onset was delayed $(15,16)$. Table 1 summarizes the onset time, duration, final induced diopter, and course of time from birth for emmetropization in each animal model of form deprivation myopia, as well as the relationship between the deprivation onset time and the amblyopia severity in rhesus monkeys.

Monocular form deprivation during the period of visual development could not only result in myopia, but also amblyopia. However, the effects of brief, moderate, and longterm form deprivation periods on the visual acuity of guinea pigs have not been investigated, and little attention has been paid to whether myopia and amblyopia occur simultaneously or successively. Therefore, in this study, we established a monocular form deprivation model in guinea pigs with our own procedures, and then recorded refraction, axial length, and the amplitude of sweep visual evoked potentials (sweep VEPs) at different intervals in the deprived eyes, contralateral eyes, and normal control eyes. We found that sweep VEPs could be easily recorded in guinea pigs, as in rats (17) and mice (18). We also found that the amplitude of sweep VEPs in the deprived eye decreased initially, while the axial length of the deprived eye increased with longer duration of form deprivation. We present the following article in accordance with the ARRIVE reporting checklist (available at http:// dx.doi.org/10.21037/atm-20-5433).

\section{Methods}

\section{Experimental animals}

Twenty pigmented guinea pigs (male, 120-150 g, 2 weeks old) were obtained from the Beijing Huafukang Laboratory Animal Ltd. (Beijing, China) and randomly assigned to two groups: the monocularly form-deprived group $(n=10)$, and the normal control group $(n=10)$. Groups of five guinea pigs were raised together in a plastic cage $(15 \times 26 \times 32 \mathrm{~cm})$ under a 12 -h light-dark cycle with light provided by white LEDs (spectrum: broad-band, illuminance: 500 lux, color temperature: 4,500 K, frequency: $50-60 \mathrm{~Hz}$ ), and room temperature was maintained at $24^{\circ} \mathrm{C}$, food (guinea pig feed and fresh vegetables) and water supplemented with Vitamin C was available ad libitum. In the monocularly form-deprived group, face-masks used for monocular form deprivation were modified by latex balloons, which covered the right eye of guinea pig, leaving the left eye, nose, mouth and ears exposed (Figure 1), as shown in an earlier report (19). Light transmission through the latex balloon was 20\% (measured by TES 1330A Digital Lux Meter, Taiwan). All animals underwent sweep VEPs and biometric measurement (refraction and axial length) prior to the experiment and at 1, 4, and 8 weeks after form deprivation induction. Sweep VEPs were done with correction of the induced myopic refractive error. In the normal control group, the right eyes of all animals underwent sweep VEPs and biometric measurement (see details below) at each of intervals matching those of the form-deprived group. 
Table 1 Onset time, duration, final induced diopter and time-course from birth for emmetropization in each animal model of form deprivation myopia, and the relationship between deprivation onset time and amblyopia severity in rhesus monkeys

\begin{tabular}{|c|c|c|c|c|c|c|c|c|c|c|}
\hline \multirow{2}{*}{$\begin{array}{l}\text { Animal models } \\
\text { Onset time for myopia } \\
\text { Duration }\end{array}$} & \multicolumn{3}{|c|}{ Macaque monkey $(1,9,15,16)$} & \multicolumn{2}{|c|}{ Chick $(2,10)$} & $\frac{\text { Mouse }(3,13)}{3-4 \mathrm{w}}$ & $\frac{\text { Tree threw }(4,11)}{40-48 d}$ & \multicolumn{3}{|c|}{ Guinea pig $(5,12)$} \\
\hline & $18 \mathrm{~m}$ & & $26 \mathrm{~m}$ & & & $2 w$ & $3 w$ & $6 \mathrm{~d}$ & $11 d$ & $16 d$ \\
\hline Diopter (D) & -13.5 & & -4.5 & -12 & -1.1 & -4 & -11.3 & -3.4 & -5.8 & -5.7 \\
\hline $\begin{array}{l}\text { Time-course for } \\
\text { emmetropization }\end{array}$ & & $18 \mathrm{~m}$ & & & & $5-7 w$ & $91-99 d$ & & $3 w$ & \\
\hline Amblyopia severity & Severe & Mild & No deficit & & & & & & & \\
\hline
\end{tabular}

d, days; w, weeks; m, months.
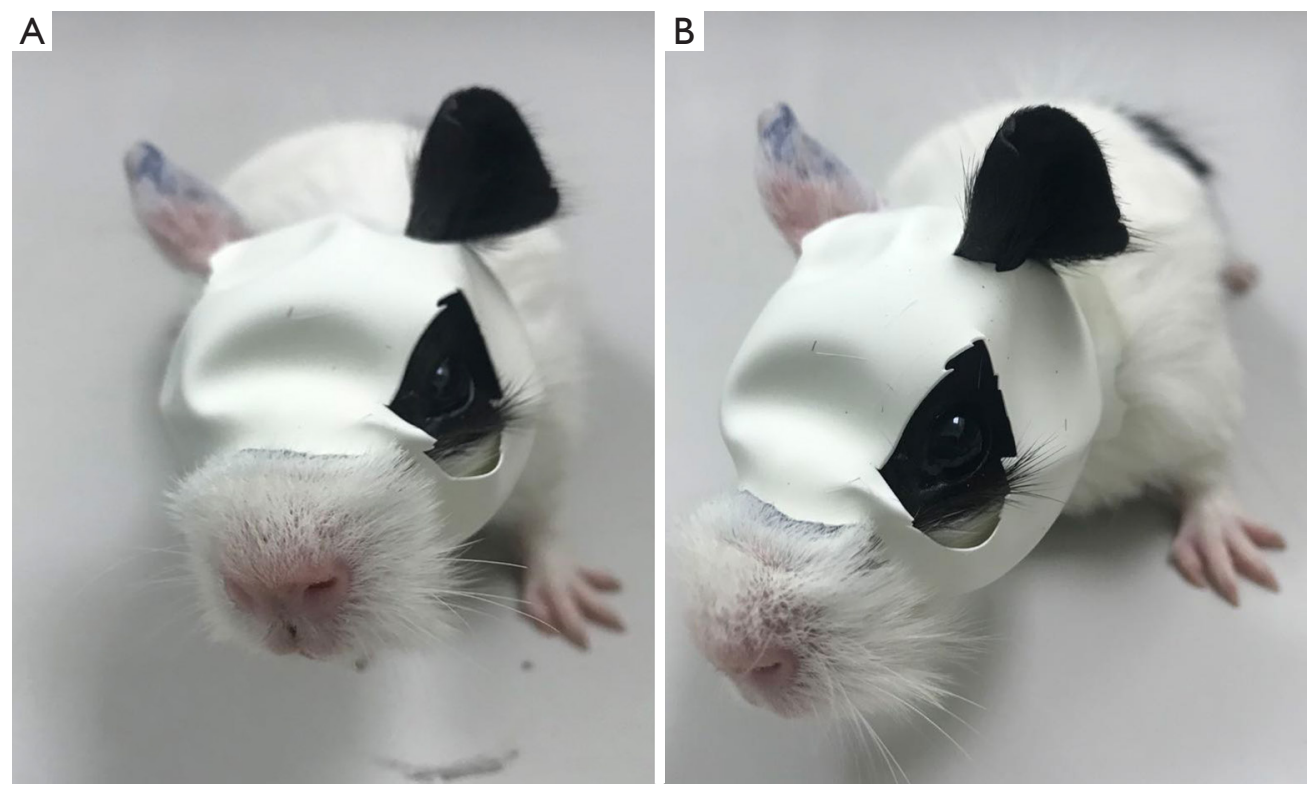

Figure 1 A pigmented guinea pig ( 2 weeks old) was well fitted with a face-mask that only covered the right eye, leaving the left eye, nose, mouth and ears exposed.

Experiments were performed under a project license (no. NKYY-DWLL-2020-099) granted by the Animal Ethical and Welfare Committees of Nankai Hospital, in compliance with the Guide for the Care and Use of Laboratory Animals of Nankai Hospital.

\section{Sweep VEPs}

\section{Visual stimulation}

Guinea pigs were anesthetized by inhaling $2 \%$ isoflurane, and mounted in a stereotaxic apparatus that restrained the snout in a fixed position (i.e., the snout was directed to the left of the screen when the right eye was stimulated). Rectal temperature was maintained at about $37.5^{\circ} \mathrm{C}$ by a water circulating warming block. When the right eye was stimulated, the face-mask was removed, and a corrective lens was employed to compensate for the induced myopia of the stimulated eye. The active needle electrode was placed in the left subcutaneous area of the binaural junction and pierced $1-1.5 \mathrm{~cm}$ forward. We put the reference electrode in the snout and the ground electrode in the hind paw, and the left eye (not stimulated) was occluded with a dark 


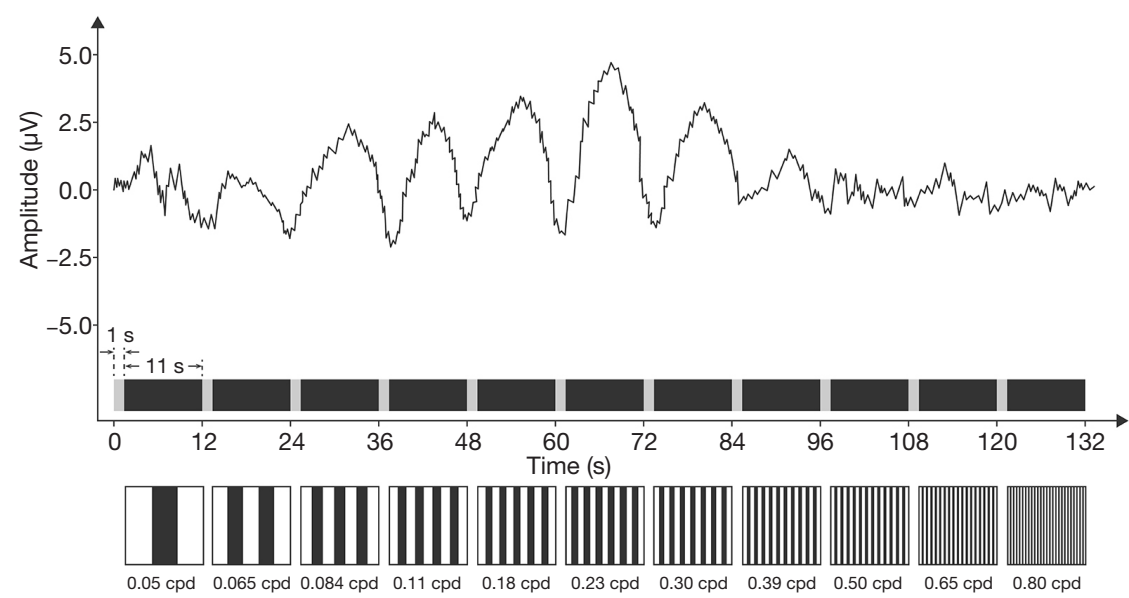

Figure 2 A diagram regarding the course of time and the 11 stimuli trace. Each spatial frequency was presented for $11 \mathrm{~s}$, and there was $1 \mathrm{~s}$ of adaptation before data collection. The stimulus contrast was $80 \%$ and the temporal reversal rate (grating wave) was $3 \mathrm{~Hz}$ (about 6 reversals/s).

patch. Reti-Port System (Roland Consult, Germany) was connected with electrodes to record sweep VEPs. The sweep VEP stimulus, a horizontally oriented sine wave grating, consisted of 11 spatial frequencies $(0.05,0.065$, $0.084,0.11,0.18,0.23,0.30,0.39,0.50,0.65$, and 0.80 cycles per degree). The stimuli were presented using a 21-inch CRT monitor. The stimulus contrast was $80 \%$ and the temporal reversal rate (grating wave) was $3 \mathrm{~Hz}$ (about 6 reversals/s). The mean luminance of the screen was $100 \mathrm{~cd} / \mathrm{m}^{2}$. The stimulus screen was viewed monocularly at $20 \mathrm{~cm}\left(100^{\circ}\right.$ wide $\times 82^{\circ}$ high $)$. Each spatial frequency was presented for $11 \mathrm{~s}$, and there was $1 \mathrm{~s}$ of adaptation before data collection. The entire sweep took $132 \mathrm{~s}(12 \mathrm{~s} \times 11$ spatial frequencies) (Figure 2).

\section{Acuity extrapolation}

Figure 3 displays an example of acuity extrapolation. The stimulus spatial frequency in cycle per degree (cpd) is plotted on the horizontal axis, and the sweep VEPs magnitude in microvolt ( $\mu v)$ is plotted on the vertical axis. The data were determined to be noise if the signal-to-noise ratio (SNR, open/filled circles) was less than 2 . As seen in Figure $3 A$, the SNR did not exceed 2 for the 0.39, 0.50, 0.65 , or $0.80 \mathrm{cpd}$ data. Acuity was determined by fitting a line between the high spatial frequency data that were above noise $(0.3 \mathrm{cpd})$ and the first spatial frequency that entered the noise $(0.39 \mathrm{cpd})$ (solid arrows). The linear fit was extrapolated to the $\mathrm{X}$-axis (zero amplitude) for visual acuity (dashed arrows). If there were no data points between the peak spatial and the noise level, the highest spatial frequency that was above noise was taken as visual acuity, as previously described $(18,20)$.

\section{Refraction}

Refraction of the eye was measured using a streak retinoscope and trial lenses in a dark room. One drop of $1 \%$ tropicamide phenylephrine (Santen, Japan) was administered to both eyes every $5 \mathrm{~min}$, three times, to achieve complete cycloplegia. The experimenter was blinded to the treatment administered. The mean value of the vertical and horizontal meridians was taken as the refraction (21).

\section{Axial length}

Axial length was measured by A-scan ultrasonography (MEDA2003s, Tianjin). The ultrasound frequency of the probe emission was $10 \mathrm{MHz}$. The conducting velocity of the anterior chamber and the vitreous chamber was $1,540 \mathrm{~m} / \mathrm{s}$, which has been previously used for measurements in guinea pigs (19). Topical anesthesia was achieved by oxybuprocaine hydrochloride (Santen, Japan), prior to the measurement. The tip of the probe had a red light that guided the probe to touch the center of the corneal surface perpendicularly during the measurement. A reliable measurement was confirmed when clear traces of various components of the eye, with consistent waves, were detected. The axial length was the distance from the cornea to the vitreous-retina interface (Figure 4).

\section{Statistical analysis}

Statistical analysis was performed using SPSS software (Version 23.0; IBM SPSS, Inc., Chicago, IL, USA). Data 

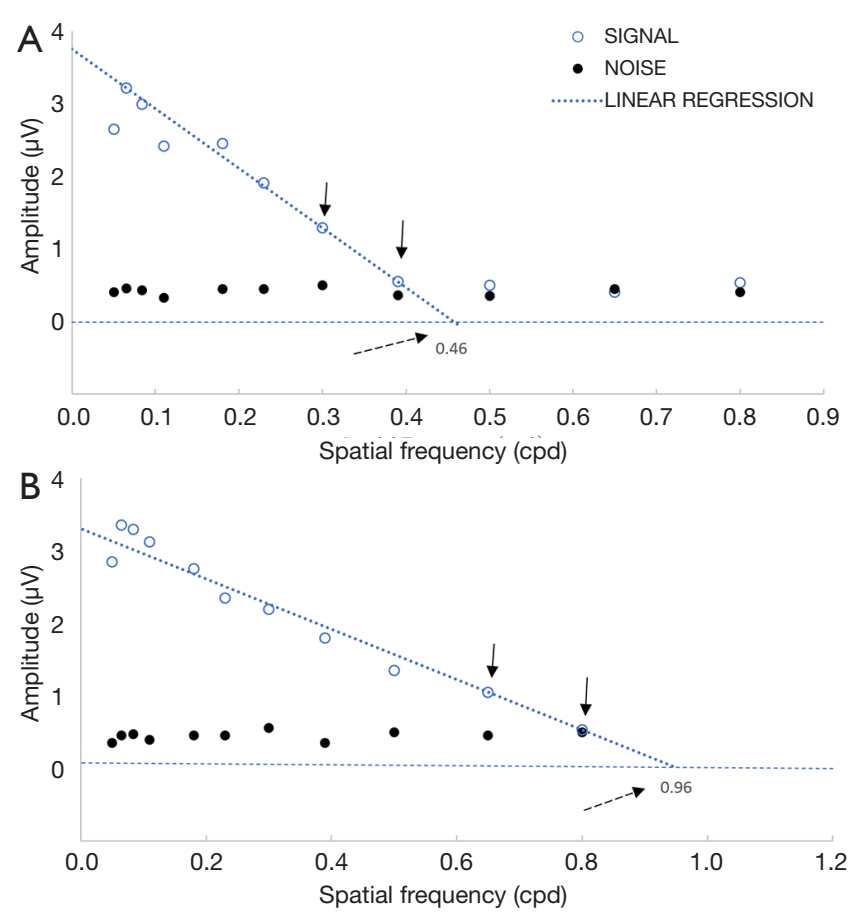

Figure 3 An example of the acuity extrapolation technique with the sweep VEPs. The stimulus spatial frequency in cycle per degree (cpd) is plotted on the horizontal axis, and the sweep VEPs magnitude in microvolt $(\mu v)$ is plotted on the vertical axis. The data were determined to be noise if the signal-to-noise ratio (SNR, open/filled circles) was less than 2. (A) Form-deprived eyes. The SNR did not exceed 2 for the $0.39,0.50,0.65$, or $0.80 \mathrm{cpd}$ data. Acuity was determined by fitting a line between the high spatial frequency data that were above noise $(0.3 \mathrm{cpd})$ and the first spatial frequency that entered the noise $(0.39 \mathrm{cpd})$ (solid arrows). The linear fit was extrapolated to the $\mathrm{X}$-axis (zero amplitude) for visual acuity (0.46 cpd, dashed arrow). (B) Normal control eyes. The SNR did not exceed 2 for the $0.80 \mathrm{cpd}$. Acuity was determined by fitting a line between the high spatial frequency data that were above noise $(0.65 \mathrm{cpd})$ and the first spatial frequency that entered the noise $(0.8 \mathrm{cpd})$ (solid arrows). The linear fit was extrapolated to the $\mathrm{X}$-axis (zero amplitude) for visual acuity $(0.96 \mathrm{cpd}$, dashed arrow). See text for details.

are presented as continuous variables and were evaluated for normality using the Shapiro-Wilk test. The results are expressed as mean \pm standard deviation (SD). All parameters among eyes and time-points were analyzed by two-way repeated measurement ANOVA followed by Bonferroni post hoc test. The differences were defined as significant at $\mathrm{P}<0.05$ and highly significant at $\mathrm{P}<0.01$.

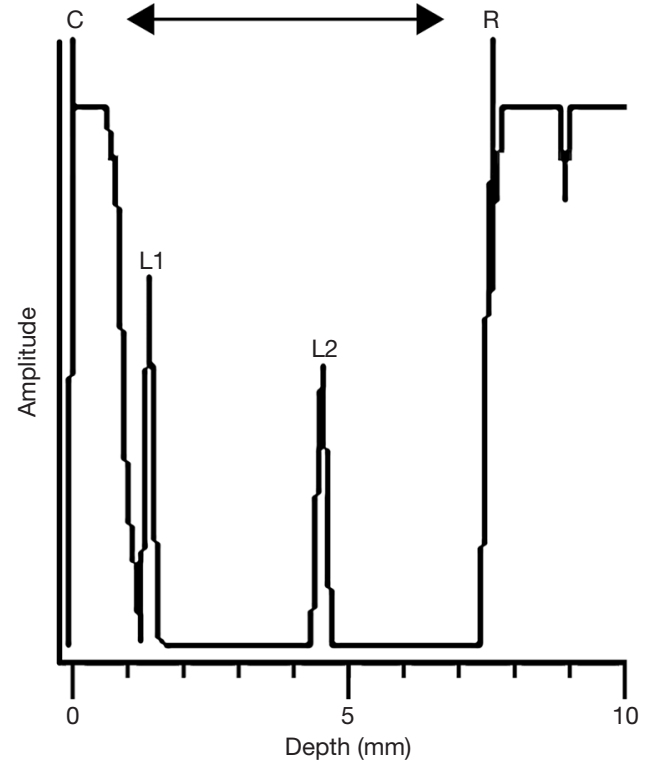

Figure 4 An example of ultrasound traces. Solid vertical lines from left to right represent front of the cornea (C), front of the lens (L1), back of the lens (L2) and the vitreous-retina interface (R). The double-headed arrow between $\mathrm{C}$ and $\mathrm{R}$ represents axial length.

\section{Results}

\section{Comparison of refraction, axial length, and acuity between different eyes}

The results obtained from measuring refraction, axial length, and visual acuity in the form-deprived eyes and the contralateral eyes of the monocularly form-deprived group, and those in the right eyes of the normal control group are presented in Table 2. The overall analysis showed that results of the comparison between groups, the comparison of times, and the interaction between groups and times were significant. Upon increasing the deprivation period, significant refractive errors were recorded in the formdeprived eyes compared with those in the contralateral and normal control eyes (Figure 5A); the axial lengths of the formdeprived eyes increased significantly compared with those in contralateral and normal control eyes after 4 weeks of form deprivation, and 8 weeks of form deprivation (Figure $5 B$ ). The changes observed in refraction and axial lengths indicate that the form deprivation myopia was established successfully during 4 weeks of form deprivation. Compared with contralateral eyes and normal control eyes, the acuity of form-deprived eyes was basically unchanged, while that of the contralateral eyes and normal control eyes improved; 
Table 2 Analysis of the interaction effects between time and treatment on refraction, axial length, and visual acuity

\begin{tabular}{|c|c|c|c|c|c|c|}
\hline Interaction effects & \multicolumn{2}{|c|}{ Refraction } & \multicolumn{2}{|c|}{ Axial length } & \multicolumn{2}{|c|}{ Visual acuity } \\
\hline Time & $1,804.794$ & 0.000 & 444.078 & 0.000 & 157.288 & 0.000 \\
\hline Time \& group & 967.353 & 0.000 & 100.469 & 0.000 & 45.731 & 0.000 \\
\hline Group & 756.869 & 0.000 & 54.515 & 0.000 & 113.336 & 0.000 \\
\hline
\end{tabular}
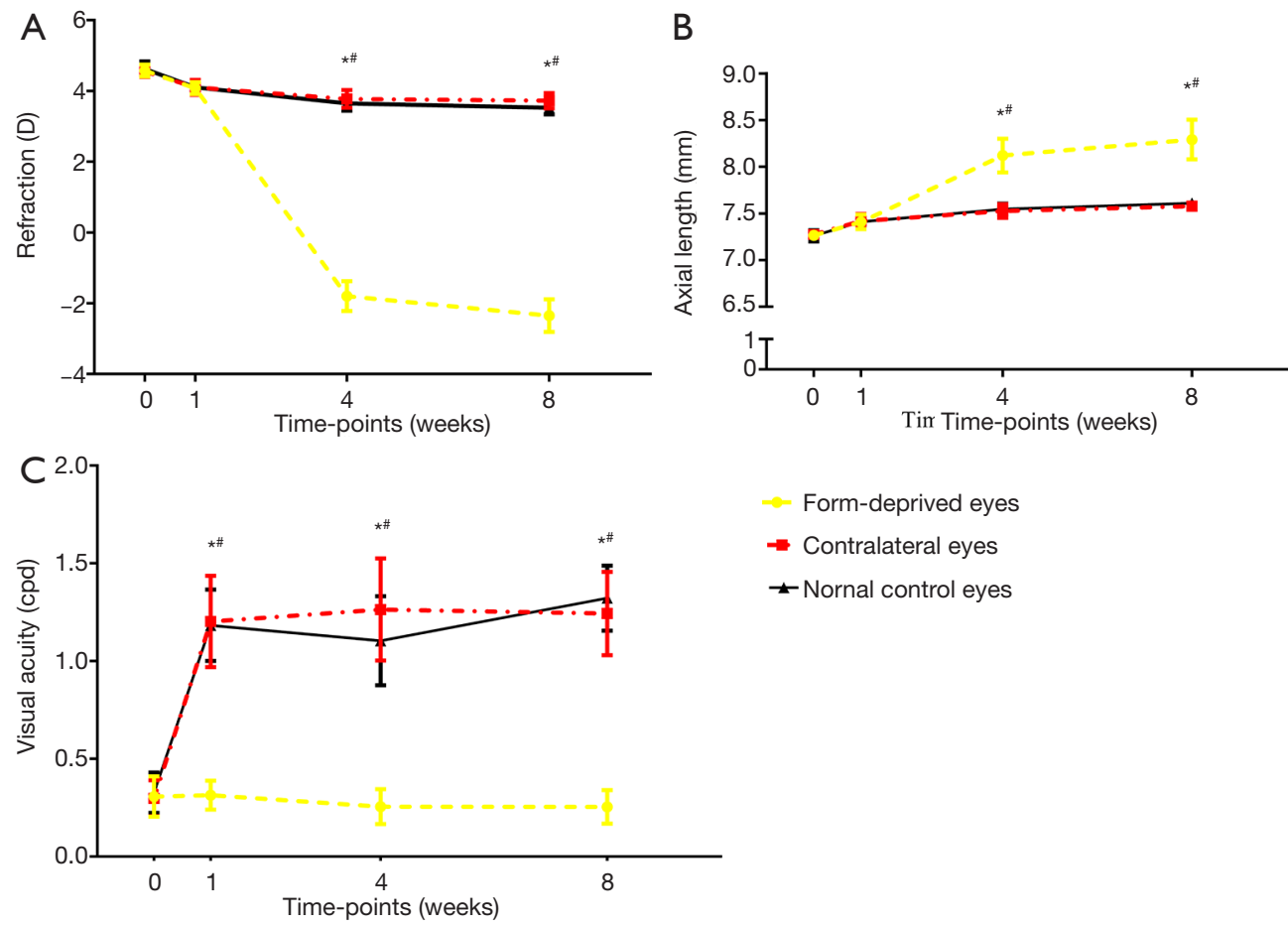

- Form-deprived eyes

- Contralateral eyes

- Nornal control eyes

Figure 5 Refraction, axial length and acuity changes in form-deprived eyes, contralateral eyes and normal control eyes at different time points. (A) Refraction at four time points; (B) axial length at four time points; (C) acuity at four time points. * $\mathrm{P}<0.01$ form-deprived eyes compared with contralateral eyes after the same duration of form deprivation; ${ }^{\#}, \mathrm{P}<0.01$ form-deprived eyes compared with normal control eyes after the same duration of form deprivation. The error bar represents mean \pm standard deviation.

there was a highly significant difference in the acuity of vision via the eyes from 1 to 8 weeks of form deprivation (Figure 5C). The results of the evaluation of acuity indicate that form deprivation amblyopia was possibly established during 1 week of form deprivation.

\section{Comparison of refraction, axial length, and acuity between different time-points in form-deprived eyes, contralateral eyes, and normal control eyes}

The overall decrease in refraction was highly significant from 0 to 8 weeks of form deprivation in form-deprived eyes $(\mathrm{P}<0.01$ between any two time-points, post hoc test); the decrease in refraction of contralateral eyes and normal control eyes was significant from 0 to 4 weeks $(\mathrm{P}<0.01$ between any two time-points, post hoc test), while there was no significant difference from 4 to 8 weeks $(\mathrm{P}>0.05$, post hoc test) (Tables 2,3). From week 0 to 1 , the diopter of both deprived eyes and open eyes decreased significantly, indicating that the guinea pig eyes had been in visual development stage. Although the refraction of all eyes decreased from 1 to 4 weeks, the decrease observed in formdeprived eyes was more obvious (Figure 5A).

Axial length of all eyes increased rapidly from 0 to 8 
Table 3 Differences of refraction, axial length and visual acuity (mean \pm SD) among all eyes in guinea pigs at each time-point

\begin{tabular}{|c|c|c|c|c|c|c|}
\hline Time-points (weeks) & 0 & 1 & 4 & 8 & $\mathrm{~F}$ & $p$ \\
\hline FD & $4.58 \pm 0.17$ & $4.08 \pm 0.17^{a}$ & $-1.80 \pm 0.42^{\mathrm{abAB}}$ & $-2.35 \pm 0.46^{\mathrm{abc} A B}$ & $2,033.568$ & 0.000 \\
\hline Contra & $4.55 \pm 0.16$ & $4.10 \pm 0.21^{a}$ & $3.78 \pm 0.25^{\mathrm{ab}}$ & $3.73 \pm 0.22^{\mathrm{ab}}$ & 81.000 & 0.000 \\
\hline Normal & $4.63 \pm 0.21$ & $4.10 \pm 0.17^{\mathrm{a}}$ & $3.65 \pm 0.21^{\mathrm{ab}}$ & $3.53 \pm 0.18^{\mathrm{ab}}$ & 88.810 & 0.000 \\
\hline$P$ & 0.646 & 0.942 & 0.000 & 0.000 & & \\
\hline \multicolumn{7}{|l|}{ Axial length (mm) } \\
\hline FD & $7.27 \pm 0.05$ & $7.41 \pm 0.08^{a}$ & $8.12 \pm 0.18^{\mathrm{abAB}}$ & $8.29 \pm 0.21^{\mathrm{abc} A B}$ & 242.408 & 0.000 \\
\hline Contra & $7.28 \pm 0.04$ & $7.42 \pm 0.08^{\mathrm{a}}$ & $7.53 \pm 0.07^{\mathrm{ab}}$ & $7.58 \pm 0.05^{\mathrm{abc}}$ & 151.070 & 0.000 \\
\hline $\mathrm{P}$ & 0.797 & 0.966 & 0.000 & 0.000 & & \\
\hline \multicolumn{7}{|l|}{ Visual acuity (cpd) } \\
\hline FD & $0.31 \pm 0.10$ & $0.31 \pm 0.07^{A B}$ & $0.26 \pm 0.09^{A B}$ & $0.25 \pm 0.09^{A B}$ & 1.805 & 0.170 \\
\hline Contra & $0.30 \pm 0.09$ & $1.20 \pm 0.23^{\mathrm{a}}$ & $1.26 \pm 0.26^{\mathrm{a}}$ & $1.24 \pm 0.21^{\mathrm{a}}$ & 79.760 & 0.000 \\
\hline Normal & $0.33 \pm 0.10$ & $1.18 \pm 0.18^{a}$ & $1.10 \pm 0.23^{\mathrm{a}}$ & $1.32 \pm 0.17^{\mathrm{abc}}$ & 115.251 & 0.000 \\
\hline $\mathrm{F}$ & 0.222 & 82.888 & 69.013 & 132.736 & & \\
\hline$P$ & 0.802 & 0.000 & 0.000 & 0.000 & & \\
\hline
\end{tabular}

"FD" refers to form-deprived eyes, "Contra" refers to contralateral eyes. ${ }^{\text {a }}$, indicates significant difference compare with 0-week time-point; ${ }^{b}$, indicates significant difference compare with 1-week time-point; ${ }^{c}$, indicates significant difference compare with 4-week time-point; ${ }^{\text {A }}$, indicates significant difference between form deprived eyes and contralateral eyes after the same duration of form deprivation; ${ }^{B}$, indicates significant difference between form deprived eyes and normal control eyes after the same duration of form deprivation.

weeks $(\mathrm{P}<0.01$ between any two time-points, post hoc test) (Tables 2,3); however, the increase observed in formdeprived eyes was steep from 1 to 4 weeks (Figure $5 B$ ).

The visual acuity at 2 weeks of age $(0$ weeks of form deprivation) was $0.31 \pm 0.10 \mathrm{cpd}$ in the form-deprived eyes; thereafter, it remained constant $(\mathrm{P}>0.05$ between any two timepoints from 0 to 8 weeks, post hoc test) and was $0.25 \pm 0.09 \mathrm{cpd}$ by 10 weeks of age ( 8 weeks of form deprivation); the acuity of the contralateral eyes increased rapidly during the first week $(\mathrm{P}<0.01,0$ vs. 1 week, post hoc test), and was $1.24 \pm 0.21$ cpd by 10 weeks of age (time-point: 8 weeks). The acuity of normal control eyes also increased rapidly during the first week $(\mathrm{P}<0.01,0$ vs. 1 week, post hoc test). Moreover, the acuity after 8 weeks of initiating the experiment was significantly different from that measured at other time points $(\mathrm{P}<0.01,8$ vs. 0 weeks; $\mathrm{P}<0.05,8$ vs. 1 weeks; $\mathrm{P}<0.01,8$ vs. 4 weeks; post hoc test) (Tables 2,3, Figure 5C).

\section{Discussion}

Monkeys, chicks, mice, tree shrews, and guinea pigs are the most widely used animal models of myopia (7); monkeys $(15,16)$ and mice $(22,23)$ have also been used in form deprivation amblyopia studies. Of the animal models used to study form deprivation myopia and amblyopia, the monkey-a non-human primate-might be an ideal animal model for both myopia and amblyopia, as the retina of monkeys has a central cone-rich region and rodrich periphery, which enables better vision. However, their availability is limited, their cost is high, and the duration of model induction is long. Mice constitute the most widely used animal model in biomedical research, with the availability of many useful transgenic strains and complete genome data. As they are mammals, their eyeball structure is basically similar to that in humans; however, the eye size of mice is very small, and a previous 
study showed that a calculated axial eye elongation of only 5.4-6.5 $\mu \mathrm{m}$ was sufficient to make the schematic eye more myopic by $1 \mathrm{D}$ (24). Therefore, it is difficult to accurately detect the small changes in eye size and refraction after myopia induction in mice (13). Guinea pigs are also mammals, and have become an increasingly popular animal model for studying myopia $(5,19,25)$. Many studies reporting the normal development of refractive state, ocular dimensions, intraocular pressure and ciliary muscle $(12,26,27)$, and the visual acuity, appear to be available (28). Although there are reports of the development of significant myopia following monocular form deprivation in many animal models, and the development of form deprivation amblyopia in monkey and mouse models, the influence of form deprivation on the acuity of eyes, and the development of form deprivation amblyopia as a result of monocular form deprivation in guinea pigs have not been investigated. The possible co-existence of amblyopia and myopia in form-deprived guinea pigs also remains unexplored. The present study was the first to use sweep VEPs to test the visual acuity of guinea pigs, and to verify the co-existence of myopia and amblyopia after monocular form deprivation in guinea pigs.

As our results showed, refraction decreased, and axial length increased in form-deprived eyes, and both were highly different from those observed in contralateral eyes and normal control eyes after 4 weeks of form deprivation. Further, acuity of form-deprived eyes remained the same as that at 2 weeks of age (0-week time-point), while that of contralateral eyes and normal control eyes improved with age. In the form-deprived eyes, the development of acuity was affected, while there was no effect on refraction and axial length after 1 week of form deprivation. The refraction and axial length changed after a longer duration of form deprivation. These results showed that visual abnormality possibly preceded ocular elongation or refraction decline; form deprivation amblyopia was developed during 1 week of form deprivation, while form deprivation myopia was established between 1 and 4 weeks of form deprivation. Eventually, amblyopia and myopia coexisted after 4 to 8 weeks of monocular form deprivation. These results were consistent with the observations of the study in which diffuser-reared infants virtually exhibited amblyopia in the treated eyes (29). In C57BL/6 mice, during the critical period, monocular form deprivation for even 4 days could induce a maximal loss of responsiveness of cortical neurons in the deprived eye (22). Even in adult mice, over 5 days of monocular deprivation could lead to an enhancement of the optokinetic response selectively through the nondeprived eye $(30,31)$. However, induction of myopia required a longer deprivation time of 2 weeks or more $(3,32)$. Therefore, we supposed that there might be an association between form deprivation myopia and amblyopia. However, the cause and effect relationship between anisometropic myopia and amblyopia is highly controversial; for example, anisometropia is usually considered to be a leading cause of amblyopia in humans, yet, there is a lack of studies that have performed longitudinal tracking of at-risk infants $(33,34)$. Studies using non-human primate models showed that anisometropia could precede amblyopia (35) or amblyopia could precede anisometropia (36), but these results were obtained from lens-reared monkey. Our research, on the other hand, is focused on form-deprived animals. Previous studies on form-deprived animals, such as monkeys $(37,38)$, chickens (39-41), tree shrews (42), and guinea pigs (43) demonstrated recovery from form deprivation myopia, even with very brief periods of unrestricted vision that were sufficient to block, or recover from, induced myopia. Similarly, the amblyopic effects of form deprivation in infant monkeys were substantially reduced by daily periods of unrestricted vision (44). A possible explanation for this recovery could be that the interruption of form deprivation failed to produce form deprivation amblyopia because the form-deprived eyes had periodically received a relatively clear image, followed by recovery from induced myopia. In addition, as described in the introduction, myopia and amblyopia induced by form deprivation mostly developed during the period of visual development. Further, monocular form deprivation caused amblyopia that resulted from ocular dominance imbalance in the visual cortex and residual suppressive binocular interactions $(45,46)$. A previous study in Macaca.arctoides monkey have indeed shown that myopia induced by form deprivation is affected by optic nerve section (47).

Based on the results and discussion above, we speculate that early brief monocular form deprivation, setting up competition between the eyes' inputs to the cortex, leads to a dramatic shift in the ocular dominance distribution of primary visual cortex units in favor of the non-deprived eye, and residual suppressive binocular interactions, resulting in amblyopia of the deprived eye (14). After the retina receives the blurred visual stimulation input of long-term duration of form deprivation, its morphological structure and contents, including a variety of bioactive substances, are changed, leading to sclera remodeling, ocular elongation, and eventually, the development of myopia. 
In this study, we did not measure the length of the anterior segment, crystalline lenses, and vitreous chamber of the eyes separately because, as shown in numerous studies of visual deprivation in animal models, there was no significant change in the anterior segment and crystalline lenses; vitreous chamber depth was significantly correlated with the total axial length of the eye. The reasons for selecting sweep VEPs to evaluate the acuity in guinea pigs are as follows. Firstly, flash stimulation is not the best stimulus for the visual cortex. Graphic stimulation provides relatively stable results, and is ideal. Secondly, rodent eyes have a short axial length, relatively thick crystalline lens, and lack fovea, but the cell density region in the retina is high. Consequently, the imaging of external objects in the retina is very small, and they are unable to achieve an effective fixation. Therefore, the moving speed of stimulation must be relatively high to bring about a change in the retinal image. Fast moving graphic stimulation can stimulate the maximum amplitude and the shortest latency of VEPs. Finally, the acuity obtained by sweep VEPs extrapolation is more intuitive, and easier to understand and deal with statistically $(17,18)$.

There are some limitations to our study. First, electrode placement might have affected the visual recording and so implanted electrode or virtual-reality optokinetic system might provide stronger reliability for repeated measurement of visual acuity (31). Second, the refraction and the axial lengths were slightly different from those reported in previous studies $(12,19)$, probably due to the influence of small eye artifacts and different A-scan ultrasonography. Third, the underlying mechanism of the visual pathway was unclear, and a future study is needed to determine the role of the retina and visual cortex. Fourth, we only used three time points to investigate changes with form deprivation. It is unclear whether the sweep VEPs changes of the deprived eyes occurred in a shorter deprivation time than 1 week, and it is also difficult to know if the myopia development occurred at 1.5 or 2 weeks after form deprivation. A further study is needed to determine the measurement changes at shorter intervals.

\section{Conclusions}

From our results alone, we cannot simply determine whether form deprivation myopia and form deprivation amblyopia occurred simultaneously or successively in guinea pigs, even though it seems likely that amblyopia occurred earlier than myopia. However, our study provides evidence that form deprivation myopia and form deprivation amblyopia could coexist in guinea pigs after a long duration of monocular form deprivation, which was induced during an early period of visual development. The axial growth, emmetropization rate, and key aspects of retinal development in guinea pigs are similar to those in humans, and there is a significant binocular interaction in the guinea pig brain (48-50). The guinea pig has potential to become an important mammalian model for studies of amblyopia. Amblyopia associated with myopic anisometropia is still difficult to treat successfully (51-54); thus, understanding the relationship of amblyopia and myopia could provide useful insight into failures of treatment.

\section{Acknowledgments}

Funding: This work was supported by the National Natural Science Foundation of China (No. 81170828; 81670837); the Tianjin Science \& Technology Foundation (No. 15JCZDJC35300); and the Tianjin Health and Family Planning Communication Foundation (No. 14KG133).

\section{Footnote}

Reporting Checklist: The authors have completed the ARRIVE reporting checklist. Available at http://dx.doi. org/10.21037/atm-20-5433

Data Sharing Statement: Available at http://dx.doi. org/10.21037/atm-20-5433

Peer Review File: Available at http://dx.doi.org/10.21037/ atm-20-5433

Conflicts of Interest: All authors have completed the ICMJE uniform disclosure form (available at http://dx.doi. org/10.21037/atm-20-5433). The authors have no conflicts of interest to declare.

Ethical Statement: The authors are accountable for all aspects of the work in ensuring that questions related to the accuracy or integrity of any part of the work are appropriately investigated and resolved. Experiments were performed under a project license (no. NKYYDWLL-2020-099) granted by the Animal Ethical and Welfare Committees of Nankai Hospital, in compliance with the Guide for the Care and Use of Laboratory Animals of Nankai Hospital. 
Open Access Statement: This is an Open Access article distributed in accordance with the Creative Commons Attribution-NonCommercial-NoDerivs 4.0 International License (CC BY-NC-ND 4.0), which permits the noncommercial replication and distribution of the article with the strict proviso that no changes or edits are made and the original work is properly cited (including links to both the formal publication through the relevant DOI and the license). See: https://creativecommons.org/licenses/by-nc-nd/4.0/.

\section{References}

1. Wiesel TN, Raviola E. Myopia and eye enlargement after neonatal lid fusion in monkeys. Nature 1977;266:66-8.

2. Wallman J, Turkel J, Trachtman J. Extreme myopia produced by modest change in early visual experience. Science 1978;201:1249-51.

3. Schaeffel F, Burkhardt E, Howland HC, et al. Measurement of refractive state and deprivation myopia in two strains of mice. Optom Vis Sci 2004;81:99-110.

4. Norton TT, Rada JA. Reduced extracellular matrix in mammalian sclera with induced myopia. Vision Res 1995;35:1271-81.

5. Howlett MH, McFadden SA. Form-deprivation myopia in the guinea pig (Cavia porcellus). Vision Res 2006;46:267-83.

6. Troilo D, Smith EL, Nickla DL, et al. IMI - Report on Experimental Models of Emmetropization and Myopia. Invest Ophthalmol Vis Sci 2019;60:M31-M88.

7. Schaeffel F, Feldkaemper M. Animal models in myopia research. Clin Exp Optom 2015;98:507-17.

8. Chakraborty R, Read SA, Vincent SJ. Understanding Myopia: Pathogenesis and Mechanisms. In: Ang M, Wong TY, editors. Updates on Myopia: A Clinical Perspective. Singapore: Springer Singapore, 2020:65-94.

9. Bradley DV, Fernandes A, Lynn M, et al. Emmetropization in the rhesus monkey (Macaca mulatta): birth to young adulthood. Invest Ophthalmol Vis Sci 1999;40:214-29.

10. Wallman J, Adams JI, Trachtman JN. The eyes of young chickens grow toward emmetropia. Invest Ophthalmol Vis Sci 1981;20:557-61.

11. Norton TT, McBrien NA. Normal development of refractive state and ocular component dimensions in the tree shrew (Tupaia belangeri). Vision Res 1992;32:833-42.

12. Zhou X, Qu J, Xie R, et al. Normal development of refractive state and ocular dimensions in guinea pigs. Vision Res 2006;46:2815-23.

13. Pardue MT, Stone RA, Iuvone PM. Investigating mechanisms of myopia in mice. Exp Eye Res 2013;114:96-105.

14. Kiorpes L. Understanding the development of amblyopia using macaque monkey models. Proc Natl Acad Sci U S A 2019;116:26217-23.

15. Harwerth RS, Smith EL 3rd, Crawford ML, et al. Behavioral studies of the sensitive periods of development of visual functions in monkeys. Behav Brain Res 1990;41:179-98.

16. Smith EL 3rd, Harwerth RS, Siderov J, et al. Prior binocular dissociation reduces monocular form deprivation amblyopia in monkeys. Invest Ophthalmol Vis Sci 1992;33:1804-10.

17. Guire ES, Lickey ME, Gordon B. Critical period for the monocular deprivation effect in rats: assessment with sweep visually evoked potentials. J Neurophysiol 1999;81:121-8.

18. Ridder WH 3rd, Nusinowitz S. The visual evoked potential in the mouse--origins and response characteristics. Vision Res 2006;46:902-13.

19. Lu F, Zhou X, Zhao H, et al. Axial myopia induced by a monocularly-deprived facemask in guinea pigs: A noninvasive and effective model. Exp Eye Res 2006;82:628-36.

20. Ridder $\mathrm{WH} 3 \mathrm{rd}$. Methods of visual acuity determination with the spatial frequency sweep visual evoked potential. Doc Ophthalmol 2004;109:239-47.

21. McFadden SA, Howlett MH, Mertz JR. Retinoic acid signals the direction of ocular elongation in the guinea pig eye. Vision Res 2004;44:643-53.

22. Gordon JA, Stryker MP. Experience-dependent plasticity of binocular responses in the primary visual cortex of the mouse. J Neurosci 1996;16:3274-86.

23. Lickey ME, Pham TA, Gordon B. Swept contrast visual evoked potentials and their plasticity following monocular deprivation in mice. Vision Res 2004;44:3381-7.

24. Schmucker C, Schaeffel F. A paraxial schematic eye model for the growing C57BL/6 mouse. Vision Res 2004;44:1857-67.

25. Lu F, Zhou X, Jiang L, et al. Axial myopia induced by hyperopic defocus in guinea pigs: A detailed assessment on susceptibility and recovery. Exp Eye Res 2009;89:101-8.

26. Ostrin LA, Wildsoet CF. Optic nerve head and intraocular pressure in the guinea pig eye. Exp Eye Res 2016;146:7-16.

27. Pucker AD, Carpenter AR, McHugh KM, et al. Guinea pig ciliary muscle development. Optom Vis Sci 2014;91:730-9.

28. Garcia MB, Jha AK, Healy KE, et al. A Bioengineering Approach to Myopia Control Tested in a Guinea Pig Model. Invest Ophthalmol Vis Sci 2017;58:1875-86. 
29. Smith EL 3rd, Hung LF. Form-deprivation myopia in monkeys is a graded phenomenon. Vision Res 2000;40:371-81.

30. Prusky GT, Alam NM, Douglas RM. Enhancement of vision by monocular deprivation in adult mice. J Neurosci 2006;26:11554-61.

31. Douglas RM, Alam NM, Silver BD, et al. Independent visual threshold measurements in the two eyes of freely moving rats and mice using a virtual-reality optokinetic system. Vis Neurosci 2005;22:677-84.

32. Pardue MT, Faulkner AE, Fernandes A, et al. High susceptibility to experimental myopia in a mouse model with a retinal on pathway defect. Invest Ophthalmol Vis Sci 2008;49:706-12.

33. Barrett BT, Bradley A, Candy TR. The relationship between anisometropia and amblyopia. Prog Retin Eye Res 2013;36:120-58.

34. Simons K. Amblyopia Characterization, Treatment, and Prophylaxis. Survey of Ophthalmology 2005;50:123-66.

35. Smith EL 3rd, Harwerth RS, Crawford ML. Spatial contrast sensitivity deficits in monkeys produced by optically induced anisometropia. Invest Ophthalmol Vis Sci 1985;26:330-42.

36. Kiorpes L, Wallman J. Does experimentally-induced amblyopia cause hyperopia in monkeys? Vision Res 1995;35:1289-97.

37. Huang J, Hung LF, Smith EL 3rd. Recovery of peripheral refractive errors and ocular shape in rhesus monkeys (Macaca mulatta) with experimentally induced myopia. Vision Res 2012;73:30-9.

38. Qiao-Grider Y, Hung LF, Kee CS, et al. Recovery from form-deprivation myopia in rhesus monkeys. Invest Ophthalmol Vis Sci 2004;45:3361-72.

39. Napper GA, Brennan NA, Barrington M, et al. The duration of normal visual exposure necessary to prevent form deprivation myopia in chicks. Vision Res 1995;35:1337-44.

40. Napper GA, Brennan NA, Barrington M, et al. The effect of an interrupted daily period of normal visual stimulation on form deprivation myopia in chicks. Vision Res

Cite this article as: Tian L, Guo YT, Ying M, Liu YC, Li X, Wang Y. Co-existence of myopia and amblyopia in a guinea pig model with monocular form deprivation. Ann Transl Med 2021;9(2):110. doi: 10.21037/atm-20-5433
1997;37:1557-64.

41. Ritchey ER, Zelinka C, Tang J, et al. Vision-guided ocular growth in a mutant chicken model with diminished visual acuity. Exp Eye Res 2012;102:59-69.

42. Siegwart JT Jr, Norton TT. The susceptible period for deprivation-induced myopia in tree shrew. Vision Res 1998;38:3505-15.

43. Zhou X, Lu F, Xie R, et al. Recovery from axial myopia induced by a monocularly deprived facemask in adolescent (7-week-old) guinea pigs. Vision Res 2007;47:1103-11.

44. Wensveen JM, Harwerth RS, Hung LF, et al. Brief daily periods of unrestricted vision can prevent form-deprivation amblyopia. Invest Ophthalmol Vis Sci 2006;47:2468-77.

45. Mitchell DE, Sengpiel F. Neural mechanisms of recovery following early visual deprivation. Philos Trans R Soc Lond B Biol Sci 2009;364:383-98.

46. Sakai E, Bi H, Maruko I, et al. Cortical effects of brief daily periods of unrestricted vision during early monocular form deprivation. J Neurophysiol 2006;95:2856-65.

47. Raviola E, Wiesel TN. An animal model of myopia. N Engl J Med 1985;312:1609-15.

48. Howlett MH, McFadden SA. Emmetropization and schematic eye models in developing pigmented guinea pigs. Vision Res 2007;47:1178-90.

49. Loeliger M, Rees S. Immunocytochemical development of the guinea pig retina. Exp Eye Res 2005;80:9-21.

50. Ates K, Demirtas S, Goksoy C. Binocular interactions in the guinea pig's visual-evoked potentials. Brain Res 2006;1125:26-30.

51. Curtin BJ, Schlossman A. Unilateral high myopia in childhood: clinical characteristics and treatment. Am Orthopt J 1976;26:65-8.

52. Kutschke PJ, Scott WE, Keech RV. Anisometropic amblyopia. Ophthalmology 1991;98:258-63.

53. Pollard ZF, Manley D. Long-Term Results in the Treatment of Unilateral High Myopia with Amblyopia. Am J Ophthalmol 1974;78:397-9.

54. Priestley BS, Hermann JS, Bloom M. Amblyopia secondary to unilateral high myopia; results of pleoptic therapy. Am J Ophthalmol 1963;56:926-32. 